\title{
Yield Prediction of some Orchard Trees Based on Soil Characteristics under Rainfed Conditions
}

\author{
Yahia I. Mohamed ${ }^{1}$, Abdalsamad A. Aldabaa ${ }^{2}$
}

\begin{abstract}
Studying the relationship between soil properties and crop yield is one of the most important tasks that should be considered under rainfed conditions. The cropping system in the north western coastal zone is mainly includes of olive and fig grown under rainfed conditions. The intercorrelation among soil properties and their influences on olive and fig yields was investigated. Therefore, multiple regression analysis was employed to generate coefficients for relative contributions of selected soil properties such as soil depth, gravel, soil texture (expressed by sand, silt, and clay), salinity, pH, and calcium carbonate on crop yield. Data of olive and fig yield were collected during 3 consecutive years of 2013, 2014, and 2015 from two different locations, namely wadi Hashem basin (7 sites) and wadi El Heriga basin ( 9 sites). The collected soil data were interpolated and mapped across the study areas. Statistically, the Pairwise comparisons of crop production demonstrated that there was a significant difference among some of the studied sites in regard to their yield potentiality. To predict the crop yield of the studied plants based on the selected soil properties, Partial Least Square Regression Model (PLSRM) was used. It depicted a profound predication model of olive and fig yield, where it produced $R^{2}$ of 0.892 with RMSE of 0.093 and $R^{2}$ of 0.995 with RMSE of 0.042 for olive and fig respectively. Eventually and for the current study, it could be concluded that most of the studied soil properties have a great influence on olive and fig yield under the rainfed condition.
\end{abstract}

Key word: soil characteristics, olive (olea europaea), yield predicition.

\section{INTRODUCTION}

In the north western coast region of Egypt, olive and fig production is enormously important for both economic and ecological reasons. Both crops are grown normally under rainfed condition, with low plant density (less than 100 trees/ha), intensive tillage, low inputs of fertilizer and pesticides and manual harvest. This region is predominated by calcareous type of soil, where a few thousands out of about 2-3 million feddan are reclaimed mainly under the dry farming agricultural systems. Future of agricultural expansion in this area demands that all soil resources should be carefully studied and evaluated with the aim of estimating their potentialities(Abd EL-Rahman et al., 1987).
Olive and fig trees are well adapted to the adverse climate conditions in the north western coastal area. For example (Sofo et al., 2008) indicated that olive trees develop a series of physiological mechanisms to tolerate drought stress and grow under harsh conditions. Similarly, Fig tree is characterized by its tolerance to water deficit. However, (Allam et al., 2007) and (AlDesouki et al., 2009) indicated that the growth and yield of fig trees were reduced under severe drought stress. Moreover, (Ouda et al., 2016) indicated that under different climate change scenarios of low rainfall in the area, olive and fig yields decreased by 79 and $44 \%$, respectively.

understanding the variability of landscape and soil characteristics and their influences on crop productivity is a vital and critical component of the site-specific and sustainable management system and land use planning, (Juhos et al., 2015). The expectable yield or productivity capacity is useful in assessing the soil suitability for agricultural use. (Sys et al., 1991) demonstrated that the relationship between crop yield and soil is very complicated and relies on the complex interactions among chemical and physical properties of soil and other external natural factors. Many studies have shown that the crop growth and yield is significantly affected by the soil characteristics. (Teka and Haftu, 2012) found that the most limiting factors that affect the olives production were soil texture and soil depth. Similarly, (Mbodj et al., 2004), in Oud Rmel Catchment of Tunisia, found that the most influential limiting factors were alkaline $\mathrm{pH}$ and the excessive amount of the soil calcium carbonate. Multiple statistical procedures have been developed to presage the crop yield, the fitness of these procedures relies on the framework and size of the database, but each method has its own limitations, (Juhos et al., 2015).

Using the stepwise multivariate linear regression analysis (SMLR), many authors have concluded that pedological parameters possess a significant relationship with crop yield (Andrews and Carroll, 2001); (Brubaker et al., 1994); (De Araujo et al., 2009); (Rezaei et al., 2006);(Adams, 1997); (Smith et al., 1993). On the other hand, simple linear regressions are usually unsuitable to describe the effect of soil indicators on the productivity. Additionally, the inter-correlation among soil properties

${ }^{1}$ Faculty of Desert and Environmental Agriculture Fuka, Alexandria, Egypt.

${ }^{2}$ Desert Research Center, Cairo, Egypt

Received August 3, 2017, Accepted September 5, 2017. 
could drive to multicollinearity problems related to the relationships between soil characteristics and crop yield. However, neglecting some variables could contribute to missing important information. Several authors have applied partial least squares regression (PLS) to overcome the multicollinearity problems between independent variables (Corwin et al., 2003); (Ping et al., 2004). Running linear combinations of variables in PLS allowed to identify soil properties that own the greatest influence on yields.

The objectives of this study were: 1) to determine the soil characteristics basically those having a significant impact on the crop productivity in the study area, consequently, the spatial variability map of each soil parameter was interpolated and 2) to develop a production function, using Partial Least Square Regression Model (PLSRM), for fig (Ficus carica L.) and Olive (Olea europaea), based on the studied soil parameters.

\section{MATERIALS AND METHODS}

\section{1- General occurrence and features}

Fig.1 showed that the study area is consisting of two wadis, namely; wadi Hashem and wadi El Heriga basins. They are located at east of Matrouh Governorate as a apart of the north west coast of Egypt. The agricultural system in these wadis is similar to other wadis and is predominately cultivated with fig and olive trees and some cereals.

Geographically, wadi Hashem basin locates between longitude $27^{\circ} 37^{\prime} 2.19^{\prime \prime}$ and $27^{\circ} 38^{\prime} 52.55^{\prime \prime} \mathrm{E}$ and latitude $31^{\circ} 07^{\prime} 34.46^{\prime \prime}$ and $31^{\circ} 10^{\prime} 10.02^{\prime \prime} \mathrm{N}$. Wadi El
Heriga basin situates between longitude $27^{\circ} 47^{\prime} 59.12^{\prime \prime}$ and $27^{\circ} 52^{\prime} 1.87^{\prime \prime} \mathrm{E}$ and latitude $31^{\circ} 04^{\prime} 0.37^{\prime \prime}$ and $31^{\circ}$ $05^{\prime} 13.68^{\prime \prime} \mathrm{N}$. The study areas as well as the northwest coastal zone are characterized by unstable rainy winter and stable warm and dry summer. The other two seasons (spring and fall) are also characterized by unstable climatic conditions, e.g., some storms during spring and occasional sudden heavy rainfall during fall. The average monthly temperature varies from 14.4 to 26.8 ${ }^{\circ} \mathrm{C}$. The wind speed ranges from 3.8 to $5.2 \mathrm{~m} / \mathrm{sec}$, and the prevailing wind is mostly from the north. However, $25 \%$ of the windy days were recorded as southerly dusty warm storms. Rainfall varies from 100 to $190 \mathrm{~mm}$ mostly falling from October to March.

The dominant geologic units in the study areas start from Tertiary Rock to Quaternary rock. The middle Miocene sediments are built up of fissured and cavernous limestone, dolomitic limestone, and sandy limestone intercalated partly with marl interbeds. Its exposures are covered by a rocky crust, which found at the top surface, on the slopes, or even in the drainage courses. The Pleistocene sediments of the Quaternary era are widely distributed in the study area and are mainly represented by oolitic limestone. The alluvial deposits are developed in the study area along the channel of the drainage lines in the form of wadi terraces and wadi fillings and in some cases, they are found at the summit of the plateau or tableland (Yousif and Baraka, 2013). These sediments are composed of sand silt, and clay with abundant carbonate grains. The thickness of alluvial deposits ranges from 0.5 to $2 \mathrm{~m}$.

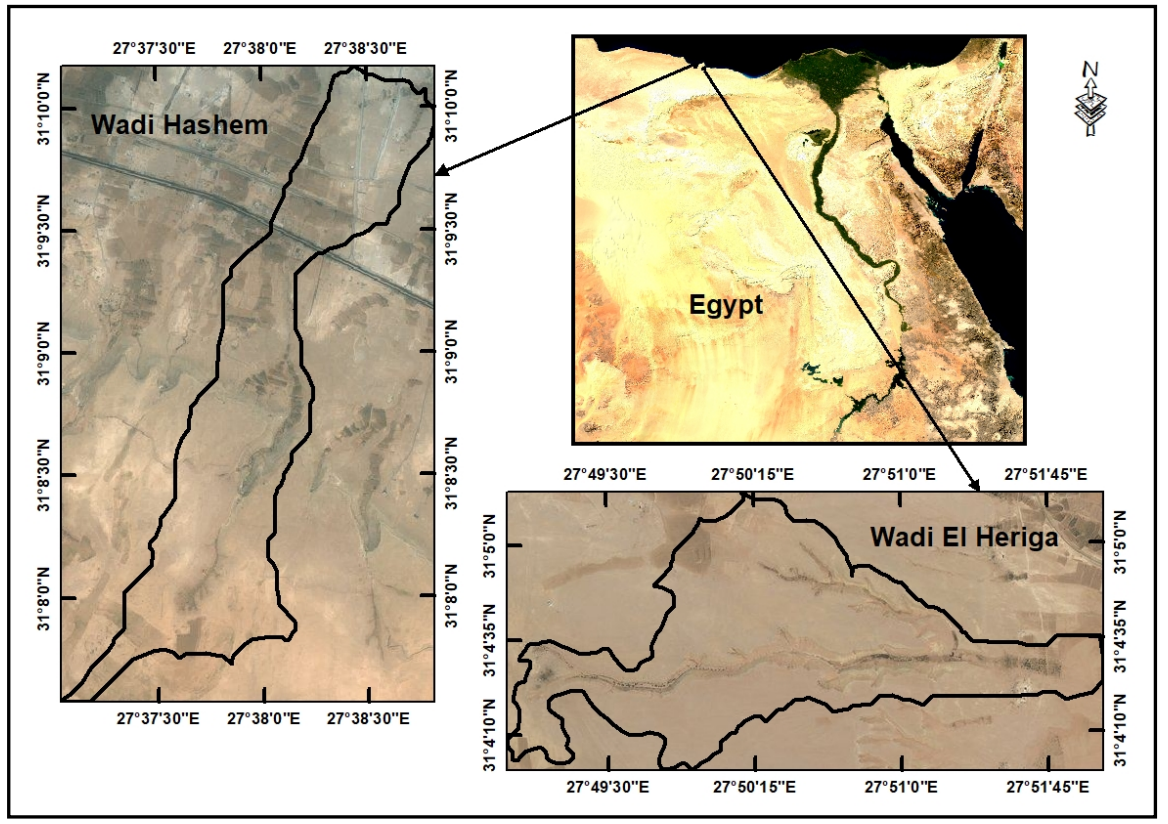

Fig. 1. Location map of the study area 
(Yousif and Baraka, 2013) demonstrated that this study area is characterized by sub-arid climatic conditions. This climate together with existing geological conditions has an effect on the present landforms. Accordingly, four main geomorphic units were established, namely; tableland (Plateau), Piedmont plain, coastal plain, and the drainage basins.

\section{2- Soil sampling and laboratory analysis}

Forty four soil samples were collected from 14 soil profiles to represent the soil condition of wadi Hashem basin. While for wadi El Heriga basin, 50 soil samples were collected from 18 soil profiles. The soil profile were demarcated randomly for each studied basin. The detailed morphological description was carried out for each soil profile using the guideline of soil description (Jahn et al., 2006). Compiled soil samples were air dried, granulated and sieved using $2 \mathrm{~mm}$ sieve. The soil texture was mechanically analyzed according to (Burt, 2004), while the gravel percentage was volumetrically determined. Based on (Burt, 2004), the electrical conductivity $(\mathrm{EC})$ was measured in $1: 2(\mathrm{~V} / \mathrm{V})$ soil water slurry using $20 \mathrm{~g}$ soil and $40 \mathrm{ml}$ distilled water and before the filtration $\mathrm{pH}$ was measured in the same slurry. The total calcium carbonate was measured using Collin's calcimeter according to(Burt, 2004).

\section{3- Mapping of soil characteristics}

As soon as the laboratory analyses ended up, the weighted average of each soil property through soil profile was calculated using the equation of SUMPRODUCT/SUM (sum of multiplying the property value by layer thickness through soil profile divided by the total depth) using MS Excel version 2007. The aim of this action was to produce a single value for each property of each soil profile representing the study areas. These single values were interpolated by the method of Inverse Distance Weight (IDW) using ArcGIS 10.4.1. However, to attain the aim of the current study, 8 soil properties i.e., soil depth, soil gravel, sand, silt, clay, soil salinity (EC), soil reaction $(\mathrm{pH})$ and lime $\left(\mathrm{CaCO}_{3}\right)$ were interpolated and mapped.

\section{4- Plant measurements}

In order to predicate or evaluate the effect of soil properties on plant productivity, each basin was considered as a sampling area. So for the plant measurements, 7 sites of wadi Hashem basin were selected; 3 for fig trees and 4 for olive trees. On the other hand, 9 sites of wadi El Heriga basin were selected; 4 for fig trees and 5 for olive trees. At each site the tree measurements such as canopy volume and crop yield were calculated from 3 replicated trees, then the average values were obtained.
The canopy volume $(\mathrm{CV})$ was calculated according to the equation: $\mathrm{CV}=0.536 \mathrm{x}(\mathrm{D})^{2} \times \mathrm{H}$; where $\mathrm{H}$ is the tree height, D1 and D2 are transversal diameters and D $=(\mathrm{D} 1+\mathrm{D} 2) / 2$, as reported by (Khabou et al., 2009). The crop yield of fig and olive trees was weighted as kg per tree and then converted to $t / F e d$. to calculate the entire production at the harvesting stage of three sequential seasons (2013, 2104 and 2015) . Moreover, the values of the suggested soil properties at each plant site were extracted from the interpolated maps in order to model the relationship between soil properties and crop yield.

\section{5- Statistical analysis}

The statistical analysis such as descriptive statistics and correlation matrix of the weighted average of soil data were performed. In order to carry out the regression model between the soil properties and the plant productivity, the soil and plant results were matched together in a spreadsheet for each plant in a separate workbook. Also, test of data normality to generate a multiple linear regression (MLR and to test model performance with correlation statistics was done. Data quality was assessed via $\mathrm{R}^{2}$, and root means square error (RMSSE). The multiple linear regression was made using partial least square regression model (PLSRM), all the previously mentioned data analyses were performed using XLSTAT statistical package,(Fahmy, 2015).

\section{RERSULTS AND DISCUSSION}

\section{1- Soil characteristics}

The total cultivated areas of wadi Hashem basin and wadi El Heriga basin are 34692 feddan (15,498 feddan in wadi Hashem basin and 19,194 feddan in wadi El Heriga basin). As shown in Tables (1), (2) and (3), the study areas are characterized by a wide variety of soil depth which ranges from $20-150 \mathrm{~cm}$ and $25-150 \mathrm{~cm}$ for wadi Hashem and El Heriga basins, respectively,. The soil gravel ranged from 0 to $38 \%$ in the soil of wadi Hashem basin and from 0 to $54.76 \%$ in wadi El Heriga basin. Depending on the weighted averages as shown in Tables (3 and 4), the gravel content ranges from 0 to $49.37 \%$ and their spatial distribution are shown in Fig. (1 and 2). The soil texture of wadi Hashem and wadi El Heriga basins ranged from sand to sandy clay loam and from sand to sandy loam, respectively. Taking the weighted averages in consideration, the soil texture was mapped using SAGA GIS, Grid Tools, analysis, Soil Texture Classification Module based on the grid of sand, silt, and clay. Accordingly, the soil texture of the study area varies from sand to sandy loam. 
Table 1. Some chemical and physical properties of Wadi Hashem Soils, NWCZ

\begin{tabular}{|c|c|c|c|c|c|c|c|c|c|}
\hline Site & $\begin{array}{l}\text { Depth } \\
\text { (cm) }\end{array}$ & $\begin{array}{c}\text { Gravel } \\
\%\end{array}$ & $\begin{array}{c}\text { Sand } \\
\%\end{array}$ & $\begin{array}{c}\text { Silt } \\
\%\end{array}$ & $\begin{array}{c}\text { Clay } \\
\%\end{array}$ & Texture & pH & $\begin{array}{c}\text { EC } \\
\text { dS/m }\end{array}$ & $\begin{array}{c}\mathrm{CaCO}_{3} \\
\%\end{array}$ \\
\hline \multirow[t]{3}{*}{1} & $0-30$ & 25.00 & 73.96 & 10.42 & 15.62 & sandy loam & 7.33 & 3.40 & 19.19 \\
\hline & $30-80$ & 35.00 & 85.04 & 6.46 & 8.50 & loamy sand & 7.30 & 3.50 & 43.62 \\
\hline & $80-130$ & 0.00 & 79.76 & 12.01 & 8.23 & loamy sand & 7.11 & 2.60 & 6.98 \\
\hline \multirow[t]{4}{*}{2} & $0-15$ & 26.50 & 81.38 & 12.41 & 6.21 & loamy sand & 7.05 & 8.70 & 22.68 \\
\hline & $15-45$ & 0.00 & 74.85 & 16.01 & 9.14 & sandy loam & 7.02 & 2.20 & 20.02 \\
\hline & $45-95$ & 36.00 & 76.88 & 9.17 & 13.95 & sandy loam & 7.25 & 1.80 & 32.28 \\
\hline & $95-145$ & 0.00 & 76.57 & 12.66 & 10.77 & sandy loam & 7.19 & 1.10 & 9.59 \\
\hline \multirow[t]{4}{*}{3} & $0-35$ & 0.00 & 77.65 & 11.23 & 11.12 & sandy loam & 7.46 & 7.30 & 18.32 \\
\hline & $35-65$ & 0.00 & 74.85 & 16.01 & 9.14 & sandy loam & 7.02 & 2.20 & 20.02 \\
\hline & $65-120$ & 0.00 & 77.52 & 10.81 & 11.67 & sandy loam & 7.08 & 3.50 & 13.96 \\
\hline & $120-150$ & 0.00 & 79.76 & 12.01 & 8.23 & loamy sand & 7.11 & 2.60 & 6.98 \\
\hline \multirow[t]{5}{*}{4} & $0-25$ & 25.00 & 78.12 & 12.65 & 9.23 & sandy loam & 7.05 & 5.20 & 22.68 \\
\hline & $25-50$ & 29.50 & 56.77 & 27.00 & 16.23 & sandy loam & 7.00 & 3.00 & 29.22 \\
\hline & $50-95$ & 32.50 & 94.00 & 3.00 & 3.00 & sand & 7.28 & 2.50 & 52.35 \\
\hline & $95-110$ & 35.00 & 85.04 & 6.46 & 8.50 & loamy sand & 7.30 & 3.50 & 43.62 \\
\hline & $110-140$ & 36.00 & 76.88 & 9.17 & 13.95 & sandy loam & 7.25 & 1.80 & 32.28 \\
\hline \multirow[t]{4}{*}{5} & $0-45$ & 0.00 & 75.76 & 16.23 & 8.01 & sandy loam & 7.53 & 3.80 & 7.85 \\
\hline & $45-80$ & 0.00 & 76.57 & 12.66 & 10.77 & sandy loam & 7.19 & 1.10 & 9.59 \\
\hline & $80-120$ & 0.00 & 83.5 & 12.13 & 4.37 & loamy sand & 7.18 & 1.30 & 8.72 \\
\hline & $120-150$ & 0.00 & 84.04 & 8.32 & 7.64 & loamy sand & 7.08 & 1.20 & 21.81 \\
\hline 6 & $0-20$ & 27.90 & 70.96 & 10.91 & 18.13 & sandy loam & 7.02 & 3.60 & 16.57 \\
\hline \multirow[t]{3}{*}{7} & $0-15$ & 0.00 & 74.10 & 13.34 & 12.56 & sandy loam & 7.39 & 0.80 & 6.10 \\
\hline & $15-45$ & 0.00 & 71.28 & 21.81 & 6.91 & sandy loam & 7.40 & 2.70 & 6.10 \\
\hline & $45-60$ & 0.00 & 79.86 & 14.13 & 6.01 & loamy sand & 7.14 & 1.90 & 2.18 \\
\hline \multirow[t]{3}{*}{8} & $0-15$ & 25.00 & 63.9 & 15.45 & 20.65 & sandy clay loam & 7.05 & 1.40 & 18.32 \\
\hline & $15-25$ & 30.50 & 80.66 & 11.81 & 7.53 & loamy sand & 7.00 & 3.60 & 4.36 \\
\hline & $25-60$ & 28.50 & 83.68 & 6.22 & 10.10 & loamy sand & 7.12 & 1.90 & 27.48 \\
\hline \multirow[t]{4}{*}{9} & $0-20$ & 0.00 & 71.78 & 13.55 & 14.67 & sandy loam & 7.47 & 3.40 & 20.06 \\
\hline & $20-40$ & 0.00 & 85.13 & 4.66 & 10.21 & loamy sand & 7.16 & 7.60 & 20.06 \\
\hline & $40-100$ & 0.00 & 79.86 & 14.13 & 6.01 & loamy sand & 7.34 & 4.30 & 12.65 \\
\hline & $100-130$ & 0.00 & 71.78 & 13.55 & 14.67 & sandy loam & 7.20 & 5.60 & 5.24 \\
\hline \multirow[t]{5}{*}{10} & $0-30$ & 25.00 & 67.72 & 10.63 & 21.65 & sandy clay loam & 7.32 & 7.80 & 21.81 \\
\hline & $30-65$ & 28.00 & 63.62 & 12.17 & 24.21 & sandy clay loam & 7.33 & 2.20 & 19.63 \\
\hline & $65-100$ & 28.00 & 76.82 & 8.17 & 15.01 & sandy loam & 7.34 & 4.30 & 12.65 \\
\hline & $100-130$ & 24.00 & 73.06 & 14.22 & 12.72 & sandy loam & 7.20 & 5.60 & 5.24 \\
\hline & $130-150$ & 25.00 & 76.09 & 13.66 & 10.25 & sandy loam & 7.00 & 1.20 & 20.06 \\
\hline \multirow[t]{4}{*}{11} & $0-20$ & 0.00 & 55.05 & 24.92 & 20.03 & sandy clay loam & 7.00 & 2.30 & 29.29 \\
\hline & $20-50$ & 31.20 & 83.16 & 12.23 & 4.61 & loamy sand & 7.00 & 4.70 & 19.19 \\
\hline & $50-80$ & 25.00 & 84.37 & 8.90 & 6.73 & loamy sand & 7.02 & 6.60 & 20.06 \\
\hline & $80-120$ & 0.00 & 73.76 & 12.23 & 14.01 & sandy loam & 7.00 & 1.70 & 24.43 \\
\hline \multirow[t]{5}{*}{12} & $0-20$ & 27.50 & 56.48 & 25.17 & 18.35 & sandy loam & 7.00 & 2.50 & 10.47 \\
\hline & $20-40$ & 34.50 & 63.96 & 16.50 & 19.54 & sandy loam & 7.00 & 4.40 & 34.9 \\
\hline & $40-80$ & 38.00 & 80.48 & 11.23 & 8.29 & loamy sand & 7.02 & 4.10 & 38.39 \\
\hline & $80-105$ & 37.00 & 81.62 & 10.87 & 7.51 & loamy sand & 7.00 & 7.24 & 33.15 \\
\hline & $105-130$ & 36.50 & 70.67 & 17.63 & 11.70 & sandy loam & 7.02 & 5.70 & 13.96 \\
\hline 13 & $0-45$ & 27.96 & 78.58 & 9.11 & 12.31 & sandy loam & 7.08 & 2.06 & 21.34 \\
\hline 14 & $0-40$ & 27.00 & 78.59 & 9.10 & 12.31 & sandy loam & 7.11 & 2.11 & 21.32 \\
\hline
\end{tabular}


Table 2. Some chemical and physical properties of Wadi El-Heriga Soils, NWCZ

\begin{tabular}{|c|c|c|c|c|c|c|c|c|c|}
\hline Site & $\begin{array}{c}\text { Depth } \\
\text { (cm) }\end{array}$ & $\begin{array}{c}\text { Gravel } \\
(\%)\end{array}$ & $\begin{array}{c}\text { Sand } \\
\%\end{array}$ & $\begin{array}{c}\text { Silt } \\
\%\end{array}$ & $\begin{array}{c}\text { Clay } \\
\%\end{array}$ & Texture & pH & $\begin{array}{c}\mathrm{EC} \\
\left(\mathrm{dSm}^{-1}\right)\end{array}$ & $\begin{array}{c}\mathrm{CaCO}_{3} \\
\%\end{array}$ \\
\hline \multirow[t]{5}{*}{47} & $0-7$ & 5.08 & 93.19 & 3.40 & 3.41 & Sand & 7.56 & 1.60 & 10.90 \\
\hline & $7-30$ & 4.61 & 82.78 & 7.12 & 10.10 & Loamy sand & 7.62 & 8.40 & 23.99 \\
\hline & $30-80$ & 2.60 & 78.06 & 13.82 & 8.12 & Loamy Sand & 7.70 & 1.42 & 10.90 \\
\hline & $80-130$ & 9.58 & 80.93 & 6.93 & 12.14 & Sandy loam & 7.58 & 4.60 & 2.18 \\
\hline & $130-150$ & 8.82 & 83.50 & 10.50 & 6.00 & Loamy sand & 7.44 & 0.70 & 17.45 \\
\hline \multirow[t]{3}{*}{48} & $0-25$ & 3.12 & 91.51 & 4.24 & 4.25 & Sand & 7.88 & 1.34 & 5.45 \\
\hline & $25-50$ & 0.00 & 82.57 & 8.52 & 8.91 & Loamy sand & 7.50 & 0.95 & 32.71 \\
\hline & $50-130$ & 5.10 & 85.68 & 8.31 & 6.01 & Loamy sand & 7.18 & 3.30 & 13.08 \\
\hline \multirow[t]{4}{*}{49} & $0-15$ & 6.89 & 92.03 & 3.98 & 3.99 & Sand & 7.00 & 4.91 & 30.53 \\
\hline & $15-50$ & 13.58 & 81.46 & 6.53 & 12.01 & Sandy loam & 7.01 & 5.83 & 30.53 \\
\hline & $50-85$ & 25.71 & 88.60 & 3.23 & 8.17 & Loamy sand & 7.03 & 5.85 & 30.53 \\
\hline & $85-140$ & 54.76 & 86.13 & 10.31 & 3.56 & Loamy sand & 7.05 & 7.78 & 28.35 \\
\hline \multirow[t]{2}{*}{50} & $0-15$ & 19.48 & 80.89 & 5.60 & 13.51 & Sandy loam & 7.45 & 0.88 & 30.53 \\
\hline & $15-40$ & 50.00 & 81.87 & 8.01 & 10.12 & Loamy sand & 7.18 & 0.65 & 4.36 \\
\hline \multirow[t]{2}{*}{51} & $0-15$ & 30.76 & 68.47 & 21.33 & 10.20 & Sandy loam & 7.09 & 0.89 & 9.37 \\
\hline & $15-40$ & 36.36 & 83.66 & 7.71 & 8.63 & Loamy sand & 7.08 & 1.84 & 27.26 \\
\hline \multirow[t]{3}{*}{52} & $0-25$ & 6.62 & 81.55 & 6.35 & 12.10 & Sandy loam & 7.94 & 0.68 & 19.63 \\
\hline & $25-75$ & 4.16 & 87.04 & 8.10 & 4.86 & Loamy sand & 7.10 & 0.74 & 21.81 \\
\hline & $75-150$ & 13.80 & 94.52 & 2.74 & 2.74 & Sand & 7.06 & 0.82 & 26.17 \\
\hline \multirow[t]{2}{*}{53} & $0-25$ & 16.25 & 71.57 & 16.13 & 12.30 & Sandy loam & 7.05 & 6.16 & 19.63 \\
\hline & $25-40$ & 28.94 & 77.19 & 18.20 & 4.61 & Loamy sand & 7.00 & 8.72 & 2.83 \\
\hline \multirow[t]{3}{*}{54} & $0-20$ & 4.61 & 76.30 & 15.56 & 8.14 & Sandy loam & 7.74 & 0.92 & 19.63 \\
\hline & $20-70$ & 3.33 & 80.41 & 15.36 & 4.23 & Loamy sand & 7.71 & 0.81 & 10.90 \\
\hline & $70-150$ & 27.84 & 93.13 & 1.86 & 5.01 & Sand & 7.58 & 0.70 & 23.99 \\
\hline \multirow[t]{2}{*}{55} & $0-25$ & 8.47 & 73.43 & 10.34 & 16.23 & Sandy loam & 7.81 & 0.74 & 19.63 \\
\hline & $25-40$ & 37.50 & 85.86 & 4.12 & 10.02 & Loamy sand & 7.45 & 2.40 & 28.35 \\
\hline \multirow[t]{4}{*}{56} & $0-10$ & 0.00 & 93.74 & 3.13 & 3.13 & Sand & 7.85 & 0.62 & 23.99 \\
\hline & $10-50$ & 0.00 & 81.42 & 14.30 & 4.28 & Loamy sand & 7.67 & 0.80 & 17.45 \\
\hline & $50-100$ & 0.00 & 83.88 & 10.11 & 6.01 & Loamy sand & 7.84 & 2.60 & 19.63 \\
\hline & $100-150$ & 0.00 & 76.46 & 11.21 & 12.33 & Sandy loam & 7.82 & 0.81 & 10.90 \\
\hline \multirow[t]{2}{*}{57} & $0-20$ & 16.12 & 78.76 & 11.01 & 10.23 & Sandy loam & 7.19 & 3.50 & 19.63 \\
\hline & $20-60$ & 30.00 & 79.75 & 14.20 & 6.05 & Loamy sand & 7.14 & 7.30 & 39.26 \\
\hline \multirow[t]{2}{*}{58} & $0-15$ & 17.80 & 73.77 & 20.10 & 6.13 & Sandy loam & 7.26 & 4.39 & 15.26 \\
\hline & $15-40$ & 25.60 & 80.59 & 12.00 & 7.41 & Loamy sand & 7.18 & 7.84 & 20.72 \\
\hline \multirow[t]{2}{*}{59} & $0-15$ & 27.20 & 76.61 & 11.26 & 12.13 & Sandy loam & 7.00 & 10.66 & 34.90 \\
\hline & 15.50 & 36.84 & 85.01 & 6.61 & 8.38 & Loamy sand & 7.05 & 15.67 & 39.26 \\
\hline \multirow[t]{2}{*}{60} & $0-20$ & 30.76 & 75.60 & 10.24 & 14.16 & Sandy loam & 7.32 & 0.73 & 26.17 \\
\hline & $20-40$ & 46.42 & 89.87 & 5.06 & 5.07 & Sand & 7.28 & 6.63 & 30.53 \\
\hline \multirow[t]{6}{*}{61} & $0-15$ & 5.17 & 74.47 & 16.91 & 8.62 & Sandy loam & 7.51 & 2.90 & 31.41 \\
\hline & $15-25$ & 35.61 & 93.81 & 3.09 & 3.1 & Sand & 7.38 & 1.30 & 32.71 \\
\hline & $25-55$ & 0.00 & 90.49 & 4.75 & 4.76 & Sand & 7.50 & 1.40 & 5.23 \\
\hline & $55-80$ & 4.22 & 88.87 & 5.56 & 5.57 & Sand & 7.30 & 1.40 & 12.21 \\
\hline & $80-140$ & 6.66 & 94.29 & 2.85 & 2.86 & Sand & 7.29 & 1.50 & 15.26 \\
\hline & $140-150$ & 3.61 & 74.56 & 15.83 & 9.61 & Sandy loam & 7.31 & 2.50 & 4.79 \\
\hline \multirow[t]{2}{*}{62} & $0-15$ & 40.44 & 65.94 & 16.26 & 17.80 & Sandy loam & 7.02 & 2.80 & 33.59 \\
\hline & $15-45$ & 53.84 & 81.16 & 10.23 & 8.61 & Loamy sand & 7.09 & 4.70 & 41.44 \\
\hline \multirow[t]{3}{*}{63} & $0-20$ & 8.00 & 91.87 & 3.12 & 4.01 & Sand & 7.81 & 0.81 & 13.08 \\
\hline & $20-80$ & 0.00 & 93.51 & 3.29 & 3.20 & Sand & 7.77 & 0.82 & 15.26 \\
\hline & $80-120$ & 0.00 & 84.06 & 10.20 & 5.41 & Loamy sand & 7.75 & 0.83 & 17.45 \\
\hline 64 & $0-25$ & 33.33 & 78.10 & 10.15 & 12.60 & Sandy loam & 7.70 & 5.51 & 19.63 \\
\hline
\end{tabular}


Table 3. The weighted average of some soil characteristics of the study area

\begin{tabular}{|c|c|c|c|c|c|c|c|c|c|}
\hline Site & $\begin{array}{c}\text { Depth } \\
\text { cm }\end{array}$ & $\begin{array}{c}\text { Gravel } \\
\%\end{array}$ & $\begin{array}{c}\text { Sand } \\
\%\end{array}$ & $\begin{array}{c}\text { Silt } \\
\%\end{array}$ & $\begin{array}{c}\text { Clay } \\
\%\end{array}$ & Texture & pH & $\begin{array}{c}\text { EC } \\
\text { dS/m }\end{array}$ & $\begin{array}{c}\mathrm{CaCO}_{3} \\
\%\end{array}$ \\
\hline \multicolumn{10}{|c|}{ Wadi Hashem } \\
\hline 1 & 130 & 31.25 & 80.89 & 7.95 & 11.17 & sandy loam & 7.23 & 4.46 & 34.46 \\
\hline 2 & 145 & 15.16 & 76.82 & 12.12 & 8.88 & sandy loam & 7.16 & 2.36 & 20.93 \\
\hline 3 & 150 & 0.00 & 77.46 & 12.19 & 11.06 & sandy loam & 7.16 & 3.95 & 14.79 \\
\hline 4 & 140 & 31.64 & 79.89 & 10.7 & 9.41 & loamy sand & 7.18 & 3.03 & 37.69 \\
\hline 5 & 150 & 0.00 & 79.67 & 12.72 & 7.61 & loamy sand & 7.27 & 1.98 & 11.28 \\
\hline 6 & 120 & 27.9 & 70.96 & 10.91 & 18.13 & sandy loam & 7.02 & 3.6 & 16.57 \\
\hline 7 & 60 & 0.00 & 74.13 & 17.77 & 8.1 & sandy loam & 7.33 & 2.03 & 5.12 \\
\hline 8 & 60 & 27.96 & 78.23 & 9.46 & 12.31 & sandy loam & 7.08 & 2.06 & 21.34 \\
\hline 9 & 125 & 0.00 & 78.95 & 9.73 & 11.32 & sandy loam & 7.24 & 4.5 & 14.43 \\
\hline 10 & 150 & 26.20 & 71.07 & 11.54 & 17.39 & sandy loam & 7.26 & 4.36 & 15.62 \\
\hline 11 & 120 & 14.05 & 75.64 & 13.51 & 10.84 & sandy loam & 7.01 & 3.78 & 22.84 \\
\hline 12 & 130 & 35.37 & 72.58 & 15.35 & 12.07 & sandy loam & 7.01 & 4.81 & 27.85 \\
\hline 13 & 45 & 27.96 & 78.58 & 9.46 & 12.31 & sandy loam & 7.08 & 2.06 & 21.34 \\
\hline 14 & 40 & 27.00 & 78.59 & 9.46 & 12.31 & sandy loam & 7.11 & 2.11 & 21.32 \\
\hline \multicolumn{10}{|c|}{ Wadi El Heriga } \\
\hline 47 & 150 & 6.18 & 81.17 & 9.57 & 9.26 & loamy sand & 7.61 & 3.39 & 10.87 \\
\hline 48 & 130 & 3.74 & 86.20 & 7.57 & 6.23 & loamy sand & 7.38 & 2.47 & 15.39 \\
\hline 49 & 140 & 32.07 & 86.21 & 6.92 & 6.87 & loamy sand & 7.03 & 6.50 & 29.67 \\
\hline 50 & 40 & 38.56 & 81.50 & 7.11 & 11.39 & loamy sand & 7.28 & 0.74 & 14.17 \\
\hline 51 & 40 & 34.26 & 77.96 & 12.82 & 9.22 & sandy loam & 7.08 & 1.48 & 20.55 \\
\hline 52 & 150 & 9.39 & 89.87 & 5.13 & 5.01 & sand & 7.22 & 0.77 & 23.63 \\
\hline 53 & 40 & 21.01 & 73.68 & 16.91 & 9.42 & sandy loam & 7.03 & 7.12 & 13.33 \\
\hline 54 & 150 & 16.57 & 86.65 & 8.19 & 5.17 & loamy sand & 7.64 & 0.77 & 19.05 \\
\hline 55 & 40 & 22.99 & 79.65 & 7.23 & 13.13 & sandy loam & 7.63 & 1.57 & 23.99 \\
\hline 56 & 150 & 0.00 & 81.41 & 11.13 & 7.46 & loamy sand & 7.79 & 1.39 & 16.43 \\
\hline 57 & 60 & 25.37 & 79.42 & 13.14 & 7.44 & loamy sand & 7.16 & 6.03 & 32.72 \\
\hline 58 & 40 & 22.68 & 78.03 & 15.04 & 6.93 & loamy sand & 7.21 & 6.55 & 18.67 \\
\hline 59 & 50 & 33.95 & 82.49 & 7.91 & 9.60 & loamy sand & 7.04 & 14.17 & 37.95 \\
\hline 60 & 40 & 38.59 & 82.74 & 7.65 & 9.62 & loamy sand & 7.30 & 3.68 & 28.35 \\
\hline 61 & 150 & 6.16 & 87.56 & 7.13 & 5.31 & loamy sand & 7.36 & 1.76 & 13.65 \\
\hline 62 & 45 & 49.37 & 76.09 & 12.24 & 11.67 & sandy loam & 7.07 & 4.07 & 38.82 \\
\hline 63 & 120 & 1.33 & 90.09 & 5.13 & 4.78 & sand & 7.77 & 0.82 & 15.63 \\
\hline 64 & 25 & 33.33 & 78.10 & 9.30 & 12.60 & sandy loam & 7.70 & 5.51 & 19.63 \\
\hline
\end{tabular}

Table 4. Descriptive statistics (Quantitative data)

\begin{tabular}{|c|c|c|c|c|c|c|c|c|}
\hline Statistic & Depth cm & Gravel \% & Sand $\%$ & Silt \% & Clay \% & pH & EC dS/m & $\mathrm{CaCO}_{3} \%$ \\
\hline Nbr. of observations & 32 & 32 & 32 & 32 & 32 & 32 & 32 & 32 \\
\hline Minimum & 25.00 & 0.00 & 70.96 & 5.13 & 4.78 & 7.01 & 0.74 & 5.12 \\
\hline Maximum & 150.00 & 49.37 & 90.09 & 17.77 & 18.13 & 7.79 & 14.17 & 38.82 \\
\hline 1st Quartile & 43.75 & 6.18 & 77.30 & 7.85 & 7.46 & 7.08 & 1.93 & 15.24 \\
\hline Median & 110.00 & 24.18 & 79.19 & 9.65 & 9.51 & 7.22 & 3.21 & 19.41 \\
\hline 3rd Quartile & 140.00 & 31.75 & 81.75 & 12.36 & 11.77 & 7.34 & 4.40 & 23.72 \\
\hline Sum & 3005.00 & 660.04 & 2552.28 & 332.99 & 313.14 & 232.54 & 112.82 & 662.81 \\
\hline Mean & 93.91 & 20.63 & 79.76 & 10.41 & 9.79 & 7.27 & 3.53 & 20.71 \\
\hline Variance (n) & 2129.27 & 195.65 & 23.69 & 9.99 & 10.03 & 0.05 & 6.81 & 64.02 \\
\hline Variance $(n-1)$ & 2197.96 & 201.97 & 24.46 & 10.31 & 10.36 & 0.06 & 7.03 & 66.09 \\
\hline Standard deviation (n-1) & 46.88 & 14.21 & 4.95 & 3.21 & 3.22 & 0.23 & 2.65 & 8.13 \\
\hline Skewness (Pearson) & -0.08 & -0.14 & 0.34 & 0.46 & 0.59 & 0.94 & 2.11 & 0.71 \\
\hline Kurtosis (Pearson) & -1.74 & -1.11 & -0.29 & -0.41 & 0.40 & -0.22 & 6.13 & 0.06 \\
\hline
\end{tabular}



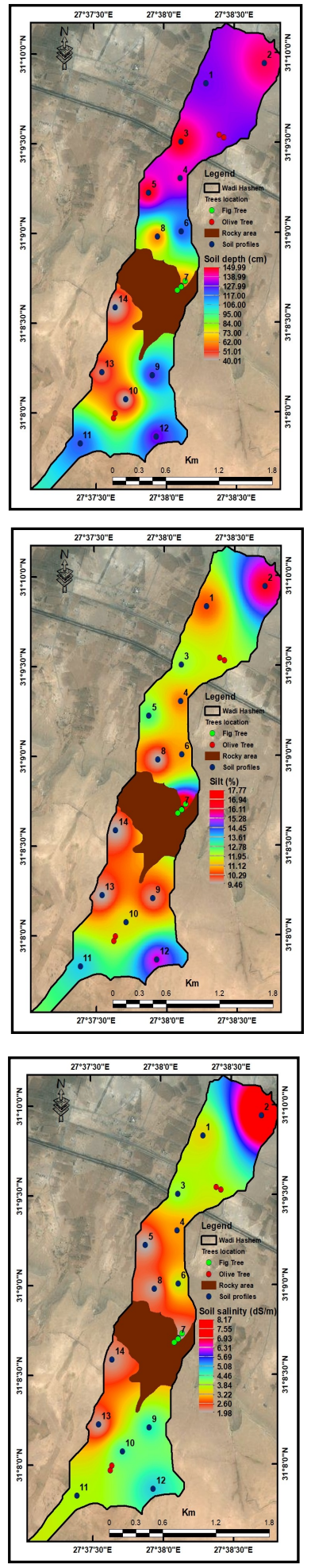
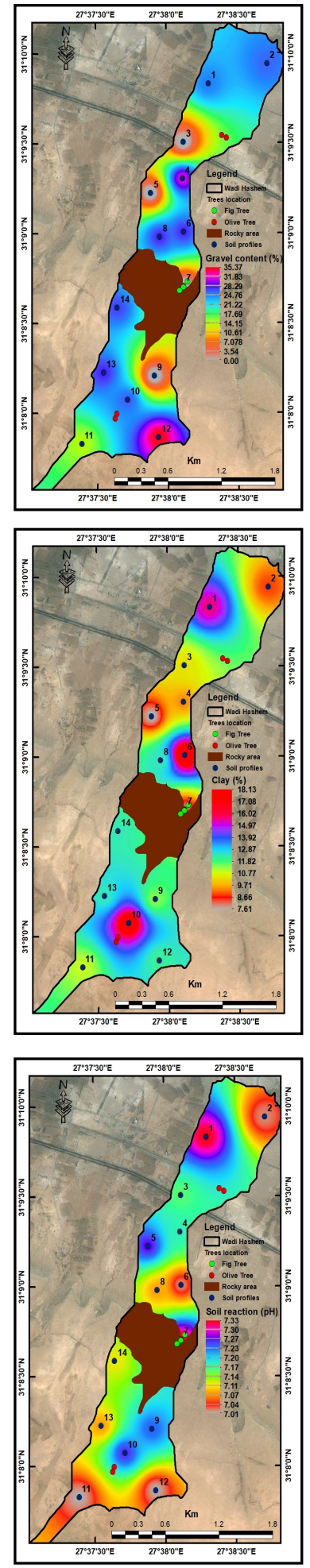
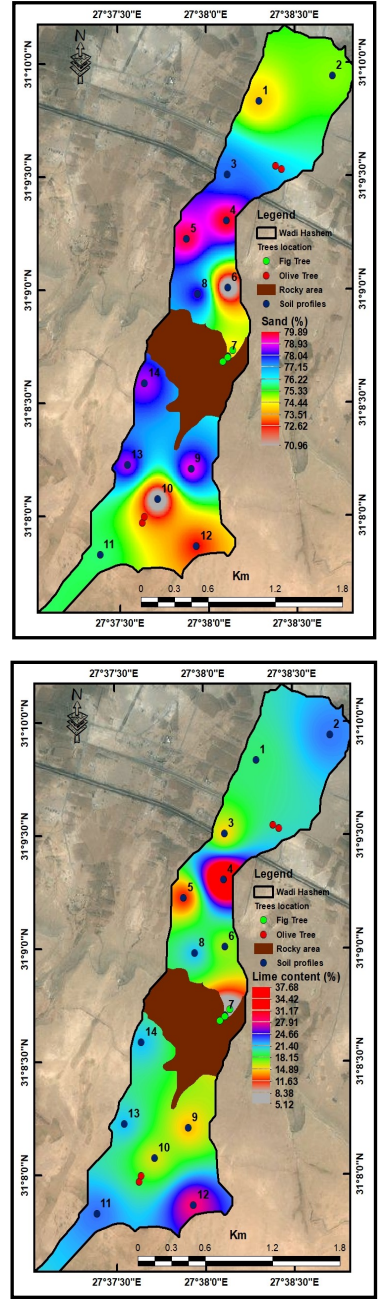

Fig. 2. Spatial distribution of some soil properties of wadi Hashem soils 

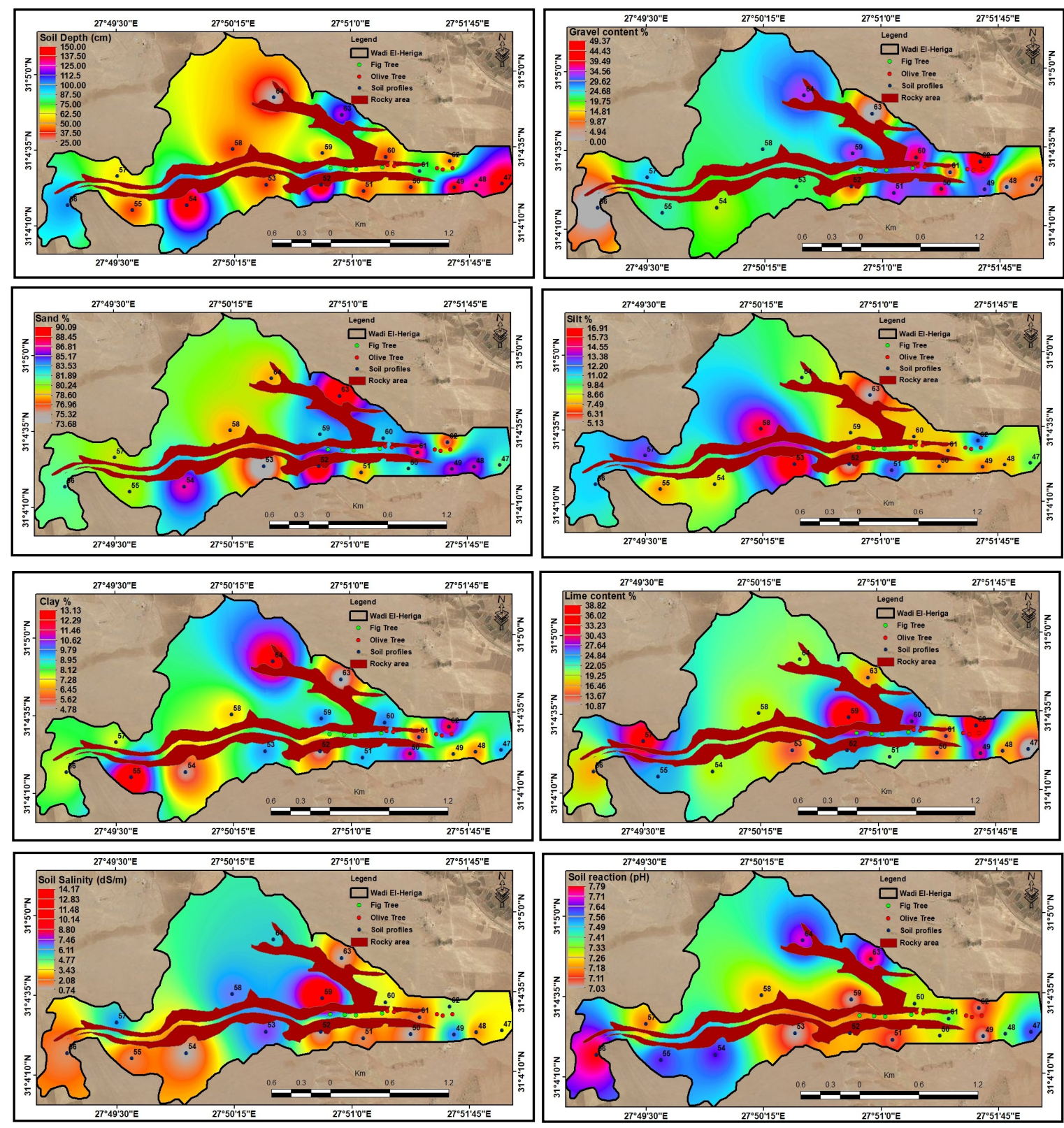

Fig. 3. Spatial distribution of some soil properties of wadi El Heriga soils

The soil reaction $(\mathrm{pH})$ fluctuated from 7.00 to 7.73 in the soil of wadi Hashem basin and from 7.00 to 7.88 in the soil of wadi El Heriga basin. The spatial variability of the $\mathrm{pH}$ was mapped based on the weighted average indicating that the soil reaction differs from 7.01 as natural soils to 7.79 as slightly alkaline soils (Fig. 1 and 2).

According to (Jahn et al., 2006), the soil salinity expressed as EC ranged from slightly saline $(0.8 \mathrm{dS} / \mathrm{m})$ to very strongly saline $(8.7 \mathrm{dS} / \mathrm{m})$ in the soils of wadi Hashem basin. While the soil salinity of wadi El Heriga basin ranged from none saline $(0.26 \mathrm{dS} / \mathrm{m})$ to extremely saline $(15.67 \mathrm{dS} / \mathrm{m})$. As shown in Table (4) and Fig (1 and 2), the weighted averages of soil salinity fluctuated from $0.74 \mathrm{dS} / \mathrm{m}$ as slightly saline to $14.17 \mathrm{dS} / \mathrm{m}$ as very strongly saline in El Heriga soils. As for the soil carbonate (lime), of wadi Hashem basin, it varied widely from 5.24 to $52.35 \%$ (moderately calcareous to extremely calcareous as described by (Jahn et al., 2006). Accordingly, the spatial variability of calcium carbonate was mapped for each area in Fig (1 and 2). 
Table 5. The soil data, plant volume, and plant yield at the examined olive location

\begin{tabular}{ccccccccccc}
\hline Location & $\begin{array}{c}\text { Depth } \\
\text { cm }\end{array}$ & $\begin{array}{c}\text { Gravels } \\
\mathbf{\%}\end{array}$ & $\begin{array}{c}\text { Sand } \\
\mathbf{\%}\end{array}$ & $\begin{array}{c}\text { Silt } \\
\mathbf{\%}\end{array}$ & $\begin{array}{c}\text { Clay } \\
\mathbf{\%}\end{array}$ & $\begin{array}{c}\text { Salinity } \\
\mathbf{d S} / \mathbf{m}\end{array}$ & $\mathbf{p H}$ & $\begin{array}{c}\mathbf{C a C O}_{\mathbf{3}} \\
\mathbf{\%}\end{array}$ & $\begin{array}{c}\text { canopy } \\
\text { volume }^{\mathbf{3}}\end{array}$ & $\begin{array}{c}\text { Yield } \\
\text { t/Fed. }\end{array}$ \\
\hline S13 & 46.66 & 35.52 & 82.81 & 7.86 & 9.34 & 3.43 & 7.29 & 26.34 & 1.00 & $0.36 \mathrm{~d}$ \\
S12 & 52.72 & 32.33 & 83.05 & 7.91 & 9.05 & 3.10 & 7.28 & 24.07 & 1.14 & $0.43 \mathrm{~cd}$ \\
S07 & 72.58 & 31.06 & 81.74 & 9.35 & 8.91 & 3.42 & 7.19 & 26.79 & 1.38 & $0.49 \mathrm{bcd}$ \\
S20 & 69.31 & 37.24 & 80.08 & 10.17 & 9.75 & 3.87 & 7.14 & 30.99 & 1.35 & $0.52 \mathrm{bcd}$ \\
S08 & 64.24 & 41.75 & 78.65 & 10.92 & 10.44 & 4.10 & 7.11 & 34.18 & 1.57 & $0.56 \mathrm{bcd}$ \\
S19 & 69.26 & 23.35 & 73.67 & 11.83 & 14.55 & 3.95 & 7.17 & 18.58 & 1.86 & $0.69 \mathrm{abcd}$ \\
S05 & 61.31 & 24.03 & 73.15 & 11.67 & 15.23 & 4.02 & 7.20 & 17.78 & 2.71 & $0.96 \mathrm{abc}$ \\
S06 & 131.65 & 16.53 & 76.61 & 11.83 & 11.56 & 3.74 & 7.19 & 20.27 & 2.91 & $1.03 \mathrm{ab}$ \\
S09 & 133.01 & 15.68 & 76.67 & 11.80 & 11.54 & 3.71 & 7.19 & 20.05 & 3.42 & $1.21 \mathrm{a}$ \\
\hline
\end{tabular}

Table 6. The soil data, plant volume, and plant yield at the examined fig location

\begin{tabular}{ccccccccccc}
\hline Location & $\begin{array}{c}\text { Depth } \\
\text { cm }\end{array}$ & $\begin{array}{c}\text { Gravels } \\
\mathbf{\%}\end{array}$ & $\begin{array}{c}\text { Sand } \\
\mathbf{\%}\end{array}$ & $\begin{array}{c}\text { Silt } \\
\mathbf{\%}\end{array}$ & $\begin{array}{c}\text { Clay } \\
\mathbf{\%}\end{array}$ & $\begin{array}{c}\text { Salinity } \\
\mathbf{d S} / \mathbf{m}\end{array}$ & $\mathbf{p H}$ & $\begin{array}{c}\mathbf{C a C O}_{\mathbf{3}} \\
\mathbf{\%}\end{array}$ & $\begin{array}{c}\text { Plant volume } \\
\mathbf{m}^{\mathbf{3}}\end{array}$ & $\begin{array}{c}\text { Yield } \\
\text { ton }\end{array}$ \\
\hline S17 & 48.75 & 24.22 & 84.51 & 7.62 & 7.87 & 6.97 & 7.16 & 28.73 & 2.46 & $1.48 \mathrm{c}$ \\
S16 & 60.06 & 26.22 & 83.23 & 8.60 & 8.17 & 4.86 & 7.19 & 25.47 & 3.16 & $1.90 \mathrm{bc}$ \\
S03 & 61.77 & 28.74 & 82.24 & 9.21 & 8.56 & 3.68 & 7.20 & 23.90 & 3.32 & $1.99 \mathrm{bc}$ \\
S04 & 62.02 & 34.66 & 82.70 & 8.05 & 9.25 & 3.37 & 7.28 & 25.78 & 3.48 & $2.09 \mathrm{bc}$ \\
S01 & 63.45 & 1.25 & 74.26 & 17.37 & 8.38 & 2.08 & 7.32 & 5.94 & 3.73 & $2.24 \mathrm{abc}$ \\
S18 & 73.32 & 0.04 & 74.13 & 17.76 & 8.11 & 2.03 & 7.33 & 5.14 & 4.86 & $2.92 \mathrm{ab}$ \\
S02 & 83.94 & 1.29 & 74.23 & 17.37 & 8.40 & 2.08 & 7.32 & 5.93 & 5.57 & $3.34 \mathrm{a}$ \\
\hline
\end{tabular}

The soils of wadi El Heriga basin behaved the same pattern described in the soils of wadi Hashem basin where lime content ranges from 2.18 to $39.26 \%$. The weighted averages of soil carbonate expressed as minimum and maximum are 5.12 and 38.82, respectively.

\section{2- Partial Least Square Regression model (PLSRM)}

Samples from olive and fig sites were subjected the PLSRM analysis in order to know the effect of some soil characteristics on the productivity of olive and fig under the rainfed condition of the north western coastal zone of Egypt. The soil data, as well as the tree measurements of the examined sites of olive and fig, are presented in tables 6 and 7, respectively.

First, it is necessary to depict all Pairwise comparisons for olive and fig locations. Based on the data shown in Table (5), There were a significant differences among the tested sites in regard to the olive yield, the highest fruit production of $1.21 \mathrm{t} / \mathrm{fed}$. was recorded at S09, while the lowest production of 0.36 $\mathrm{t} /$ fed. was at S13. a significant difference was recorded between S09 and locations number S08, S07, S20, S12 and S13 which is mainly due to the difference in all of the soil characteristics with exception remarked for salinity and $\mathrm{pH}$ measurements.

All Pairwise comparisons for fig sites are shown in Table (6) and demonstrated that there is a clear significant difference of fig production between site No. 2 and sites Nos. 4, 03, 16 and 17. It is clear from Table
(6) that the difference among these sites resulted from the variation of all of the examined soil data except clay and $\mathrm{pH}$ in the two sites. According to Table 7, site 2 recorded the highest yield of fig (3.34 t/fed), while site 17 attained the lowest value of production that was down to $1.48 \mathrm{t} /$ fed.

The data shown in table (7) describe the correlation between soil properties and olive and fig yield. Accordingly, there is a positive correlation between olive yield and soil depth, silt, clay, and salinity. In contrary, a negative correlation was found with gravel content, sand, $\mathrm{pH}$, and $\mathrm{CaCO}_{3}$. The good relationship among the studied soil characteristics was extracted in the form of the following equation that produced a $\mathrm{R}^{2}$ of 0.892 and RMSE of 0.093 , Fig (4).

Olive yield $=1.48571+0.00314 *$ Depth $0.00914 *$ Gravels $-0.00918 *$ Sand $+0.03213 *$ Silt +

$0.00729 *$ Clay $+0.01542 *$ Salinity $-0.04413 * \mathrm{pH}-$ $0.00863 * \mathrm{CaCO}_{3}$

Whereas the output from the linear combination of variables in PLSRM, stated by (Ping et al., 2004), allows identifying soil properties that have the greatest influence on yield. Accordingly, the current study indicated that most influencing soil properties could be arranged in the order of; gravel, depth, silt, sand, carbonate, and clay, while salinity and $\mathrm{pH}$ are not having significant influences. In this sense the obtained data are agreed with which obtained by (Teka and Haftu, 2012) and (Mbodj, Mahjoub and Sghaiev, 2004) where the soil depth, soil texture, and calcium carbonate are the most 
limiting factors of olive production. The correlation presented in the Table (7) demonstrated that the soil properties such as soil depth, silt, clay, and $\mathrm{pH}$ had a positive influence on the fig yield while the other soil parameters such as gravel, sand, salinity, and $\mathrm{CaCO}_{3}$ have a negative influence on the fig yield.The following equation shows a very good relationship between the soil properties and fig yield with $\mathrm{R}^{2}$ of 0.995 and RMSE of 0.042 , Fig (4).
Fig yield $=-20.062+0.05329 *$ Depth $-0.00246 *$ gravels $+0.00035^{*}$ sand $+0.00013 *$ silt $-0.06100 *$ clay + $0.07765 *$ salinity $+2.63467 * \mathrm{pH}+0.00086 * \mathrm{CaCO} 3$

In order to distinguish the most influencing soil properties on the fig yield as outcomes from PLSRM, they could be arranged in the order of; depth, sand, carbonate, silt, $\mathrm{pH}$, salinity, and gravel, while clay content is having no significant influence.

\section{Table 7. Correlation of Olive and fig yield comparing with soil data}

\begin{tabular}{ccccccccc}
\hline Variables & Depth & Gravels & Sand & Silt & Clay & Salinity & pH & CaCO $_{3}$ \\
\hline Olive yield & 0.83 & -0.86 & -0.73 & 0.81 & 0.60 & 0.41 & -0.29 & -0.65 \\
Fig yield & 0.99 & -0.74 & -0.84 & 0.83 & 0.07 & -0.82 & 0.82 & -0.84 \\
\hline
\end{tabular}

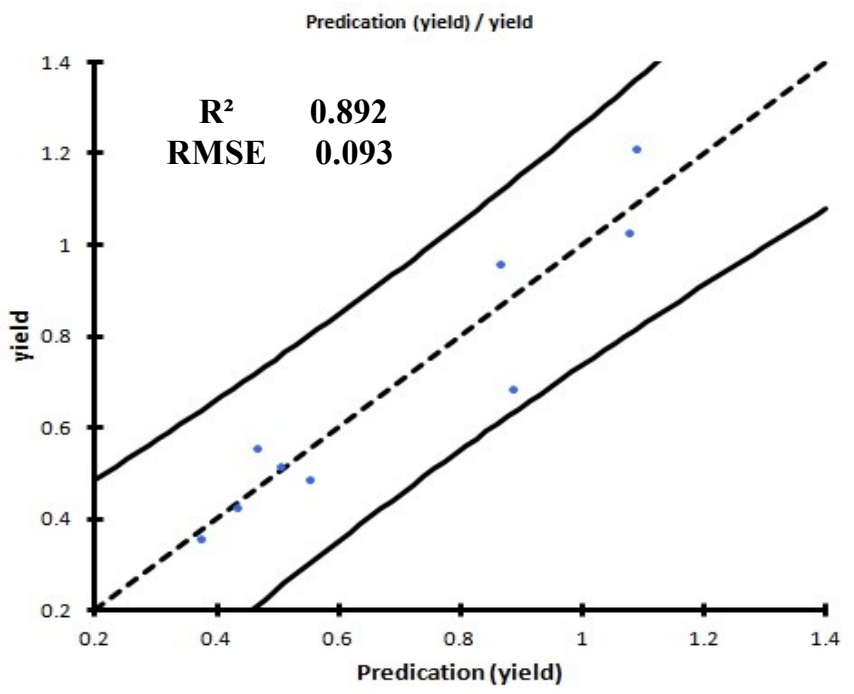

Fig .3. Partial Least Square Regression Model (Olive)

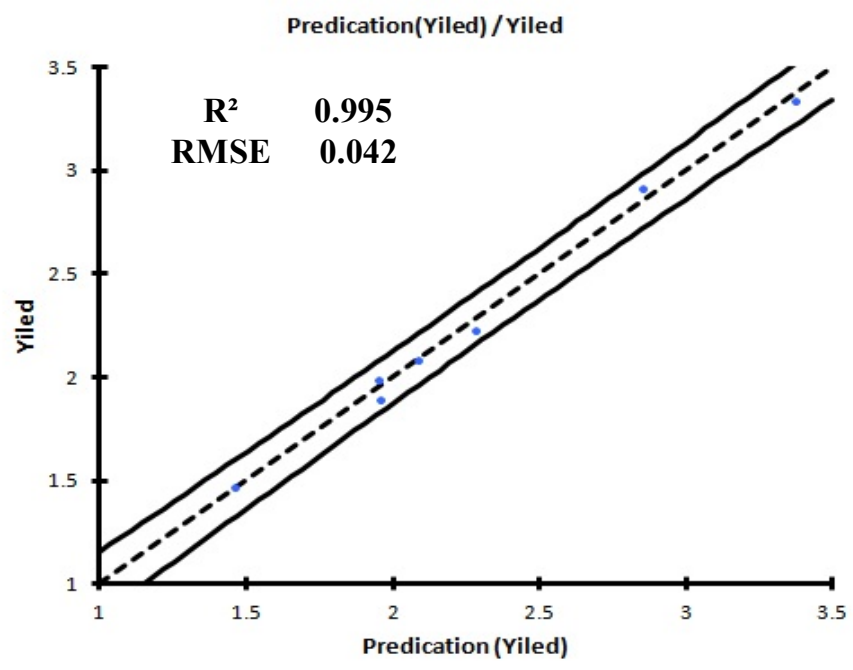

Fig.4. Partial Least Square Regression Model (Fig) 
Our study focused only on the link between tree yield and some soil parameters. However, it is well known that there are more important parameters that have a great impact on the crop yield i.e. rainfall, available nutrients, .....etc. Therefore, further studies should be focused on testing the reliability of the predictive models developed in this study. Correction also may be needed to be made for excessive soil parameters and plant measurements covering the entire study area of the NWCZ. Additionally other soil parameters should be considered which may have a profound influence on crop yield such as macro and micronutrients.

\section{CONCLUSIONS}

This study utilized a model approach called Partial Least Square Regression Model (PLSRM) to predict the crop yield of olive and fig under the rainfed conditions... The soil and plant measurements were collected from two different wadis representing the soils of the area east of Matrouh Governorate. The soil samples were subjected to standard lab determination. Accordingly, each soil parameter was interpolated for the whole study area. The extracted soil data such as soil depth, gravel, soil texture (sand, silt, and clay), $\mathrm{pH}$, salinity, and $\mathrm{CaCO}_{3}$ were used to develop PLSRM to determine the crop yield. Summarily, the current paper highlighted the use of a model for yield prediction based on soil data. The developed equation for olive and fig displayed a good relationship between soil parameters and crop yield producing $\mathrm{R}^{2}$ and RSME of 0.892 and 0.093 , for olive yield respectively. while they were 0.995 and 0.042 for fig yield. Results of this study demonstrated that some soil parameters had a negative influence while others have a positive influence on crop yield of both olive and fig.

\section{REFERENCES}

Abd EL-Rahman, S., Y. Kasim, and H. El-Attar. 1987. Soils of north western coastal region in land use classifications. Al Mağallat al-mișriyyat li-'ulum al-aradi, 17-28.

Adams, J. C. 1997. "Fortran 95 handbook: complete ISO/ANSI reference," MIT press.

Al-Desouki, M., I. A. El-Rhman, and A.Sahar. 2009. Effect of Some Antitranspirants and Supplementery Irrigation on Growth, Yield and Fruit Quality of Sultani Fig (Ficus Carica) Grown in the Egyption Western Coastal Zone under Rainfed Conditions. Research Journal of Agriculture and Biological Sciences 5, 899-908.

Allam, K., M.Adly, and M.Mourad. 2007. Effect of supplemental irrigation and intercropping treatments on the productivity of fig trees and lentil crop in the Northwest Coast. Misr Journal Agricultural Engineering 24, 88-102.
Andrews, S. S., and C. R.Carroll. 2001. Designing a soil quality assessment tool for sustainable agroecosystem management. Ecological Applications 11, 1573-1585.

Brubaker, S., A.Jones, K.Frank, and D.Lewis. 1994. Regression models for estimating soil properties by landscape position. Soil Science Society of America Journal 58, 1763-1767.

Burt, R. 2004. "Soil survey laboratory methods manual."

Corwin, D., Lesch, S., Shouse, P., Soppe, R., and Ayars, J. 2003. Identifying soil properties that influence cotton yield using soil sampling directed by apparent soil electrical conductivity. Agronomy Journal 95, 352-364.

De Araujo, J. L., L.dos Anjos, and M. G.Pereira. 2009. Soil attributes and distinction of pedoenvironments for agriculture in the MBYA Indian Reserve in Ubatuba (SP). Revista Brasileira de Ciencia do Solo 33, 1765-1776.

Fahmy, T. 2015. XLSTAT, Version 2015. Addinsoft, Paris.

Jahn, R., H.Blume, V.Asio, O.Spaargaren, and P.Schad. 2006. "Guidelines for soil description," FAO.

Juhos, K., S.Szabó, and M.Ladányi. 2015. Influence of soil properties on crop yield: a multivariate statistical approach. International Agrophysics 29, 433-440.

Khabou, W., F. B.Amar, H.Rekik, M.Beghir, and A.Touir. 2009. Performance evaluation in olive trees irrigated by treated wastewater. Desalination 246, 329-336.

Mbodj, C., I.Mahjoub, and N.Sghaiev. 2004. Land Evaluation in the Oud Rmel Catchment, Tunisia. 24th Course Professional Master. Geometric and Natural Resources Evaluation. 10th Nov 2003-23 rd Jun 2004. IAO, Florence, Italy. Istituto Agronomico per l'Oltremare IAO, Florence, Italy 21, 41-72.

Ouda, S., M.Ewise, and T.Noreldin. 2016. Projection of productivity of cultivated crops in rain-fed areas in Egypt under climate change. Cogent Food \& Agriculture 2, 1136256.

Ping, J., C.Green, K.Bronson, R.Zartman, and A.Dobermann. 2004. Identification of relationships between cotton yield, quality, and soil properties. Agronomy journal 96, 15881597.

Rezaei, S. A., R. J.Gilkes, and S. S.Andrews. 2006. A minimum data set for assessing soil quality in rangelands. Geoderma 136, 229-234.

Smith, J. L., J. J.Halvorson, and R. I.Papendick. 1993. Using multiple-variable indicator kriging for evaluating soil quality. Soil Science Society of America Journal 57, 743749.

Sofo, A., S.Manfreda, M.Fiorentino, B.Dichio, and C.Xiloyannis. 2008. The olive tree: a paradigm for drought tolerance in Mediterranean climates. Hydrology and Earth System Sciences Discussions 12, 293-301.

Sys, C., E.Debaveye, and E.van Ranst. 1991. "Land evaluation," General Administration for Development Cooperation. 
Teka, K., and M. Haftu. 2012. Land suitability characterization for crop and fruit production in Midlands of Tigray, Ethiopia. Momona Ethiopian Journal of Science 4, 64-76.
Yousif, M., and A.Baraka. 2013. Assessment of water resources in some drainage basins, northwestern coast, Egypt. Applied water science 3, 439-452.

\section{الملخص العربي}

\section{التتبؤ بانتاجية بعض المحاصيل البستانية بناء علي خصائص التربة تحت الظروف المطرية}

$$
\text { يحيي إير اهيم محمد، عبدالصمد عبدالستار علي الضبع }
$$

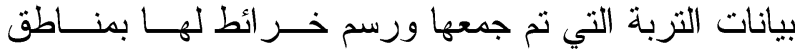
الدراسة. وقد أظهرت الدراســات الاحــــائية لمقارنــات Pairwise بعض المو اقع المدروسة. للتنبؤ بانتاجية المحــصول للتــين و الزيتون على أساس خصائص التربة المختارة، تم استخدام نموذج مربع الانحدار الجزئي Partial Least Square وبناءا

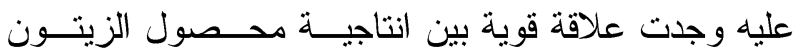
و التين وصفات التربة الطبيعية و الكيميائية، حيث كانت قيمة

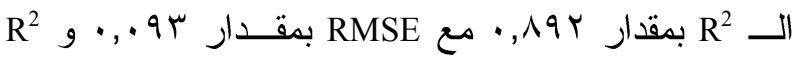

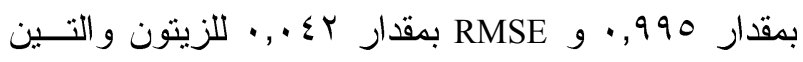
على التو الي. وخلصت الدراسة إلى أن معظم صفات التزبة المدروسة ذات تأثير ا كبير ومعنوي على انتاجية محصولي الزيتون و التين تحت ظروف الزر اعة المطرية.
ير اسة العلاقــة بــين خـصـائص التربـــة و الانتاجيــة المحصولية هي واحدة من أهم الدراسات التي ينبغي النظر

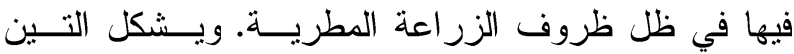
و الزيتون اغلب المساحات المنزرعــة بالــساحل الـسمالي

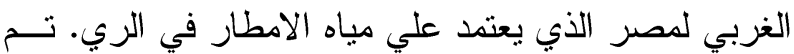

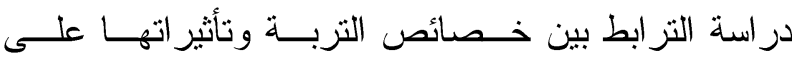

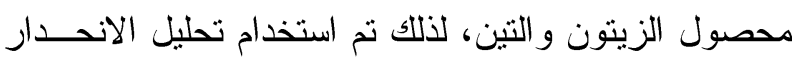

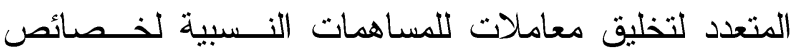
التربة المختارة مثل عمق التزبة و الحصى وقــــوام التزبـــة (التي يعبر عنها بالرمل و الطمي و الطين) و الملوحة ودرجة

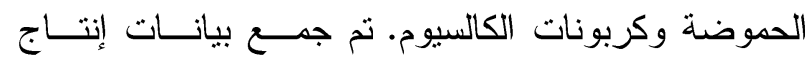

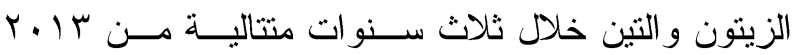

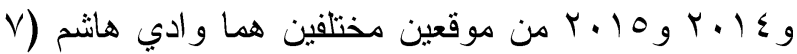
مو اقع نباتية) وو ادي الحريقة (9 مو اقع نباتية). تم اســتيفاء 